\title{
A case of atypically located left atrial myxoma with concomitant acute myocardial infarction and severe pulmonary hypertension
}

\author{
Vahit Demir a,*, Hüseyin Ede a, Sevinç Şahin ${ }^{\text {b }}$, Onur Akgün ${ }^{\text {a }}$, Yaşar Turan ${ }^{a}$, Alirıza Erbay a \\ a Bozok University, The Faculty of Medicine, Department of Cardiology, 66200 Yozgat, Turkey \\ b Bozok University, The Faculty of Medicine, Department of Pathology, 66200 Yozgat, Turkey
}

\section{A R T I C L E I N F O}

\section{Article history:}

Received 1 July 2016

Received in revised form 4 August 2016

Accepted 5 August 2016

Available online 10 August 2016

\section{Keywords:}

Myxoma

Echocardiography

Mitral stenosis

Myocardial infarction

\begin{abstract}
A B S T R A C T
Myxomas are the most frequently seen benign cardiac tumor. They mostly originate from interatrial septum. They can lead variable signs and symptoms. Opposite to their benign structure, embolic and obstructive complications can be fatal. Myxomas can rarely lead acute myocardial infarction due to coronary embolism. In this article, we presented a left atrial myxoma case that originated from posterior mitral annulus with simultaneous acute inferoposterior myocardial infarction, severe pulmonary hypertension and dynamic mitral stenosis. The patient was managed with successful percutaneous transluminal coronary angioplasty of the left circumflex artery without stenting and surgical removal of the myxoma consecutively. Pulmonary hypertension dropped significantly in postoperative follow-up.

(c) 2016 The Society of Cardiovascular Academy. Production and hosting by Elsevier B.V. All rights reserved. This is an open access article under the CC BY-NC-ND license (http://creativecommons.org/licenses/by-nc-nd/4.0/).
\end{abstract}

\section{Introduction}

Myxomas are the most frequent benign cardiac tumor but they are potentially hazardous. Seventy-five percent of myxomas are found in the left atrium (LA), mostly attached to interatrial septum (IAS). Mitral valve stenosis or systemic embolization are the most commonly seen clinical presentations of left atrial myxomas along with constitutional symptoms. Systemic embolism has been reported in 30\%-50\% of the case series. ${ }^{1}$ A majority of the cases are sporadic but $7 \%$ of them have familial backgrounds. The left atrial myxomas have a significant potential for systemic embolization perioperatively to both peripheric and coronary vasculature. ${ }^{2,3}$ Detection of this kind of pathology is very important in determining the treatment strategy since underlying pathology may lead to recurrence. Additionally, stenting with dual antiplatelet can increase bleeding risk perioperatively. In this article, we presented a case of atypically originated left atrial myxoma with simultaneous acute myocardial infarction and severe pulmonary hypertension. The patient was managed with successful percutaneous tranluminal angioplasty of the left circumflex artery (LCX) without stenting and surgical removal of the myxoma consecutively. In this article, we aimed to emphasize importance of preprocedural echocardiographic examination and the combined approach of percutaneous

\footnotetext{
* Corresponding author.

E-mail addresses: dr.vdemir@hotmail.com (V. Demir), huseyinede@gmail.com (H. Ede), sevcelik82@gmail.com (S. Şahin), onur_akgun@hotmail.com (O. Akgün), yasar044@yahoo.com (Y. Turan), alirizaerbay@gmail.com (A. Erbay).

Peer review under responsibility of The Society of Cardiovascular Academy.
}

transluminal coronary angioplasty (PTCA) and surgery for the left atrial myxoma.

\section{Case report}

The patient, a 77-year-old woman, was admitted to the emergency room with clinical presentation of acute inferoposterior myocardial infarction with a squeezing chest pain of one-hour duration radiating to the shoulders. In the history, she had no relevant prior disease or any risk factor for cardiovascular disease and she did not describe any signs of heart failure or arrhythmias previously.

Blood pressure values were $130 / 70 \mathrm{mmHg}$, the pulse rate was 72 beats/min, the oxygen saturation was normal. Lung fields were clear of rales or ronchi except for a decrease in respiratory sound at the base of the right lung on auscultation. Cardiac examination revealed apical diastolic murmur of moderate intensity without opening snap at the left lateral decubitis position.

An initial ECG revealed sinus rhythm and premature atrial beats with significant ST segment elevation in leads II, III and aVF consistent with transmural ischemia.

A transthoracic echocardiographic examination showed the presence of an echogenic, mobile mass stemming from the corner of the lower IAS and the LA and protruding toward the posterior mitral leaflet and orifice, compatible with myxoma. The mass, $36 \times 28 \mathrm{~mm}$ in size, was prolapsing toward the left ventricle and producing mitral gradient of $12 \mathrm{mmHg}$ (Fig. 1). Along with these findings, there were inferoposterior wall motion hypokinesia, the left ventricule ejection fraction (LVEF) was $48 \%$, moderate to severe tricuspid regurgitation, the estimated pulmonary artery systolic pressure (PASP) was 


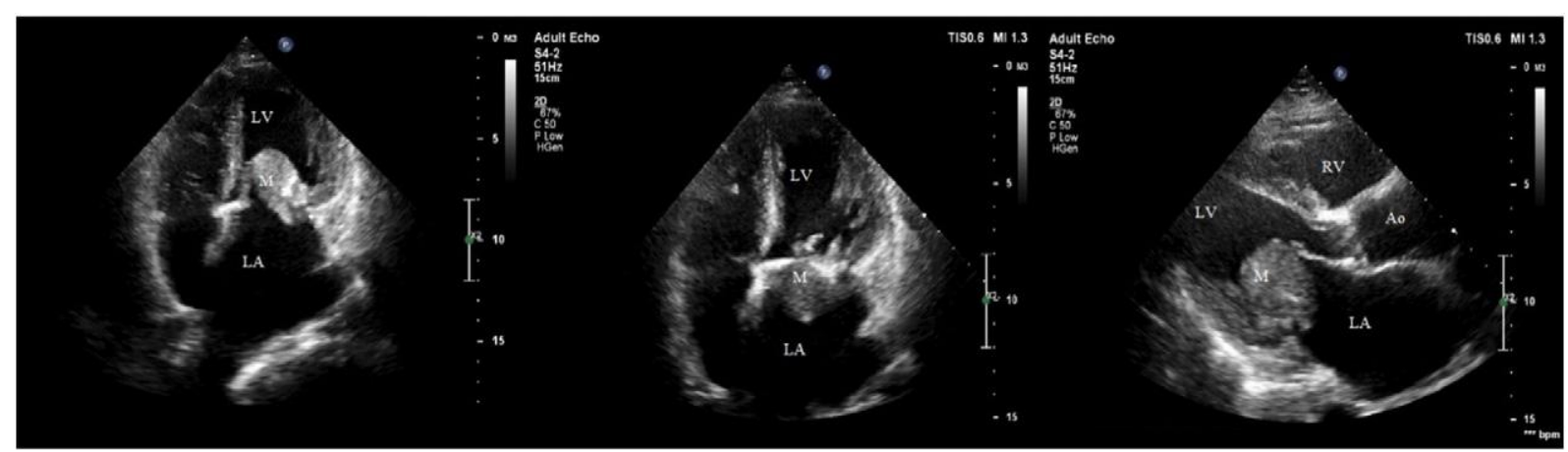

Fig. 1. Echocardiographic views of the myxoma.

$90 \mathrm{mmHg}$ and the LA diameter was $48 \mathrm{~mm}$. Following prompt antiplatelet and anticoagulant treatment, she was transferred to the coronary angiography laboratory immediately. She had thrombotic total occlusion at the distal segment of the LCx along with non-significant, stable, atherosclerotic plaques at the left anterior descending and the right coronary arteries. Successful PTCA was performed without stenting for the LCx 30 min after her admission. Three days later, she underwent operation. A tumoral lesion of $45 \times 38 \times 46 \mathrm{~mm}$ in size with stalk stemming from posterior mitral annulus was excised via left atriotomy (Fig. 2). Under a light microscope, the tumor was composed of elongated, fusiform, stellate or polygonal cells with round to ovoid nuclei immersed in a myxoid stroma. No cytological atypia was present. Immunohistochemically, the tumor cells showed positivity for vimentin, CD34, factor VIII, calretinin and S100. They were negative for pancytokeratin and CD68. Histochemically, the myxoid stroma was positive for Alcain blue and PAS. In addition, focal calcification and excessive hemorrhage were detected in the tumor, mostly in the central area. Microscopic features were diagnosed as myxoma (Fig. 3). Postoperative ECG recordings were free of ST elevations on inferior leads. Additionally, postoperative first day echocardiography revealed significant decrease in PASP to $30 \mathrm{mmHg}$ without any transmitral gradient and with no change in dimensions of the LV and the LA and mild mitral regurgitation. In the following days till discharge, the echocardiographic findings were similar to the previously described ones. The patient was discharged without complication. Postoperative 30th day echocardiographic examination showed mild mitral regurgitation and mild to moderate tricuspit regurgitation with estimated PASP of $40 \mathrm{mmHg}$ with the LVEF of 59\%.

\section{Discussion}

Myxoma is the most common benign cardiac tumor. Left atrial myxomas mostly present with changing levels of dyspnea imitating mitral stenosis and/or systemic embolization occurring in 30\% to 50\% of cases. ${ }^{1}$ The incidence of coronary embolization is $0.06 \% .{ }^{4}$ Here, we presented a case of myxoma that originated from the posterior mitral annulus with concomitant acute inferoposterior myocardial infarction.

Atrial myxomas mostly stem from the fossa ovalis portion of the interatrial septum. The next most common location for myxomas is the posterior wall of the left atrium. However, valvular involvement of myxomas is more rare. ${ }^{2}$ To the best of our knowledge, annular involvement has not been reported. In our case, the myxoma originated from the posterior mitral annulus with a pedicle. As in our case, females are more prone to have myxomas compared to males with a peak incidence at the third and sixth decades. Additionally, $67 \%$ of the subjects develop signs and symptoms of mitral stenosis in their life course, ${ }^{5}$ however, our case was asymptomatic previously. It may be due to the development of severe pulmonary hypertension in the long term leading to reduced blood flow through the pulmonary artery and low cardiac output.

Compared to computed tomography and magnetic resonance imaging, transthoracic echocardiography is the method of choice in the emergency setting. It gives functional and anatomical data related to the myxoma. Myxomas present their clinical outcomes in accordance with location, size and mobility. Thus, symptomatology can vary respectively. Our case was asymptomatic with advanced age till the admission with acute myocardial infarction. As known, the incidence of coronary embolization is very rare. ${ }^{4}$ It may be explained with the perpendicular alignment of the coronary ostia with respect to the aortic blood flow and protection of coronary ostia by the opening aortic valve leaflets. Approximately half of the cases involve the RCA. There is a concrete explanation for this tendency. There have been reports indicating that papillar or villous myxomas are more brittle and embolise more easily than myxomas with a smooth surface, it is not a must for embolization. Absence of previous coronary artery and risk factors for cardiovascular disease, absence of significant atherosclerotic coronary lesions, obtaining TIMI 3 flow just after guide wire passage and sustaining it

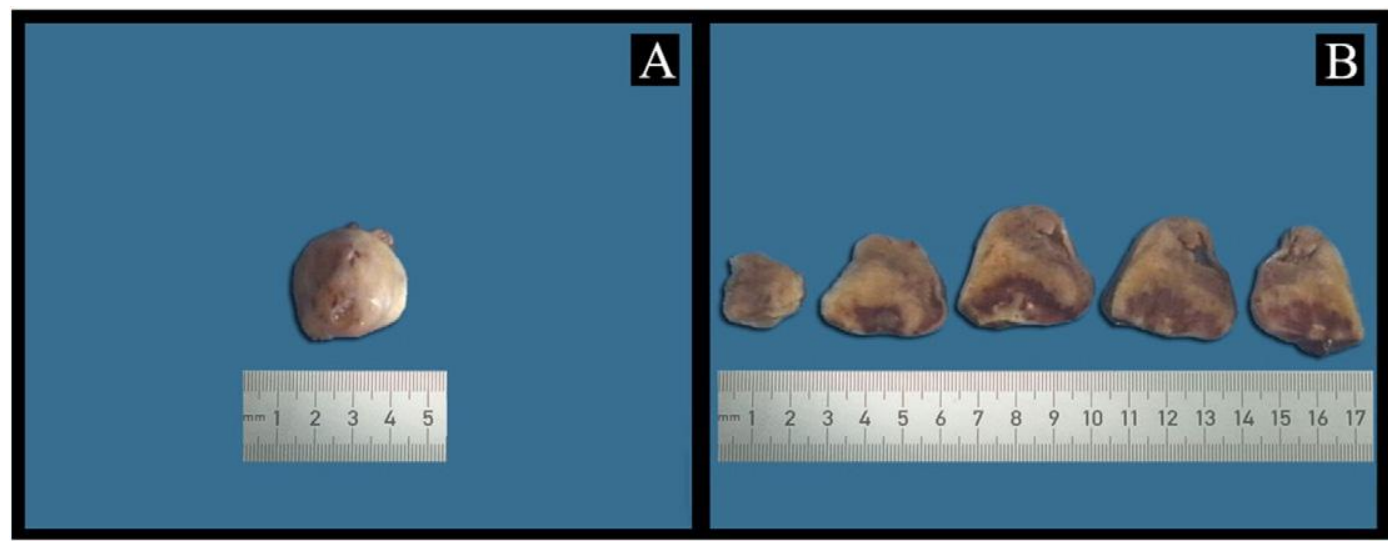

Fig. 2. Gross photographs of the lesion. (A) The rough surface of the lesion with a thin capsule. (B) The cut surfaces of the lesion containing hemorrhagic areas. 


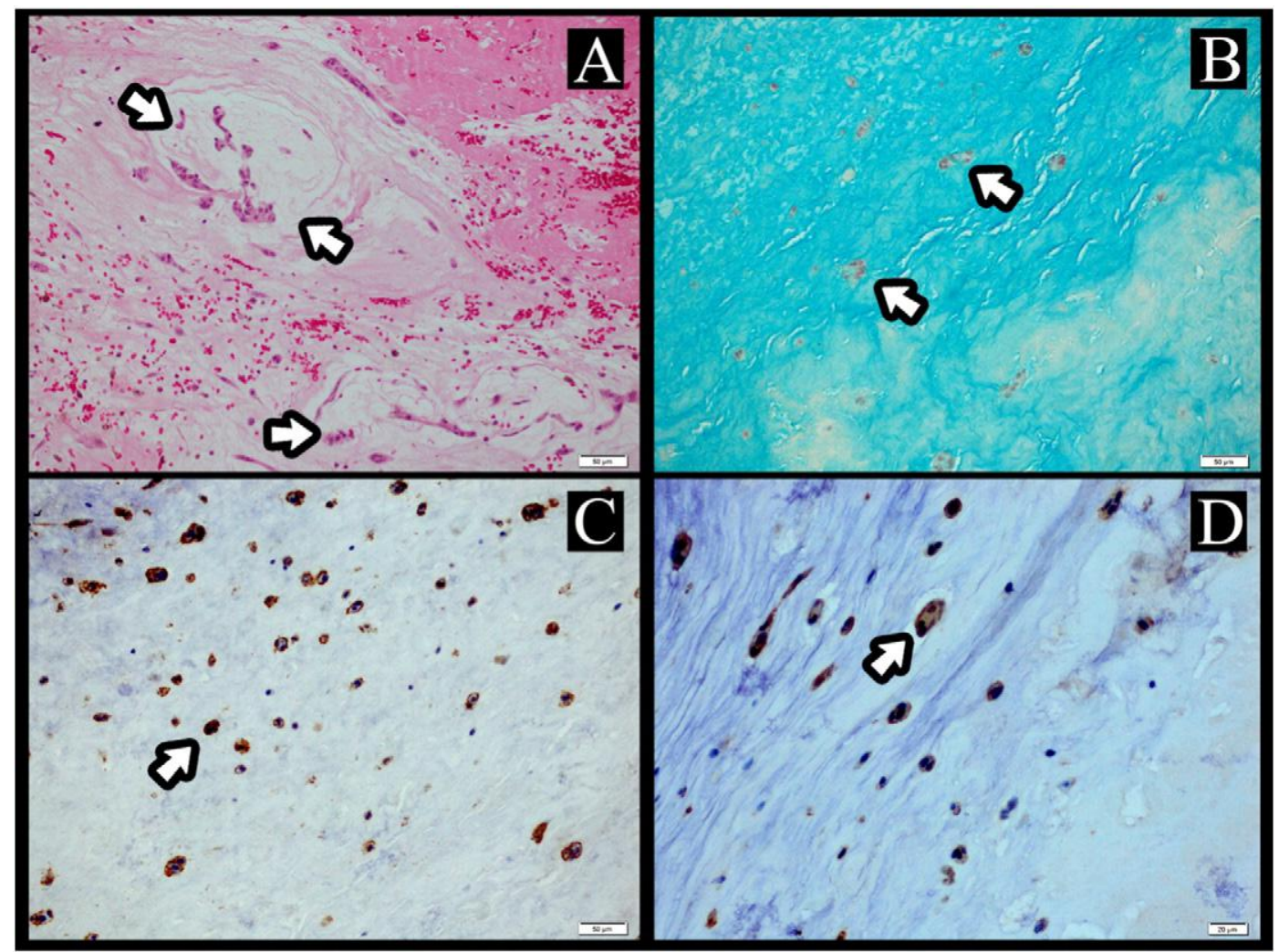

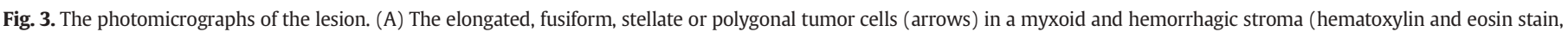

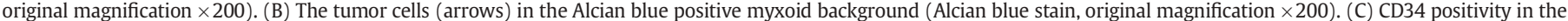

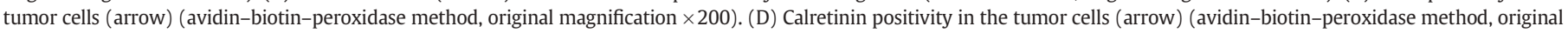
magnification $\times 400)$.

with simple PTCA without stenting were suggested coronary embolism rather than atherosclerotic etiology for acute myocardial infarction. We did not perform thrombus aspiration due to the distal location of the occlusion and obtaining TIMI 3 flow as soon as the guide wire passage through the lesion. Additionally, embolism is not the sole reason for acute myocardial infarction but reduced coronary perfusion at cardiac diastole due to reduced cardiac output by tumor-related valvular stenosis may contribute to the process. Since the patient was suffering both mechanical and embolism complication of the myxoma, we preferred the surgical excision of the myxoma. Postoperative echocardiographic examination proved the dynamic nature of myxomarelated findings. Both PASP and tricuspid regurgitation were improved respectively along with symptoms. In the literature, there have been reports implying positive correlation between myxoma size and pulmonary hypertension as in our case. ${ }^{6}$

Percutaneous coronary intervention (PCI) of acute coronary syndromes with concomitant cardiovascular problems such as congenital abnormalities, myxoma, aortic dissection, valvular diseases, infective endocarditis are challenging. Mostly due to bleeding complications or necessity of long-term dual antiplatelet limit the use of stenting in primary PCI for acute coronary syndromes. Here, we preferred to perform PTCA alone. Another important issue in the management of the myxoma is mechanical obstruction of mitral valve by the mass. It can lead to acute pulmonary edema and termination of coronary perfusion during this period. These clinical settings can further complicate the patient status. Additionally, development of new neurological findings during and after $\mathrm{PCI}$ should raise suspicions of recurrent embolism or PCI-related adverse event. Differentiation can be important during surgical management. In our case, there was not any neurological deterioration.

Histopathological diagnosis of myxoma is usually straightforward due to its characteristic features similar to our case. ${ }^{7,8}$ However, myxomas rarely include heterolog components, such as bland or atypical glandular structures (2\%), thymic rests, bone formation, chondroid tissue and extramedullary hematopoiesis (7\%) that might cause difficulty in the diagnosis of myxoma. ${ }^{8}$ It should be noted that if myxoma contains glandular structures, it might be misinterpreted as metastatic adenocarcinoma. ${ }^{8}$ Thus, the presence of a past or simultaneous history of adenocarcinoma elsewhere, presence of tumor invasion to the cardiac walls and using immunohistochemical methods for organ-specific antibodies may aid in distinguishing myxoma from metastatic adenocarcinomas. ${ }^{8}$ The lesions in our case were processed totally, but no heterolog component was detected.

In a review of 48 patients, they found that the average age of patients with concomitant acute myocardial infarction and myxoma was 46 years and anterior wall involvement was less common than inferior wall. Our case was 77 years old with inferior wall involvement. Also, coronary angiographic examination of these patients yielded normal coronary arteries in $48.8 \%$ of the subjects and obstructive coronary artery disease in $51.2 \%$ of the subjects. Culprit lesion was the LCX in $38.1 \%$, the right coronary artery in $28.6 \%$ and the left anterior descending artery in $23.8 \%$. Similar to the literature, LCx involvement was the culprit lesion in our case. ${ }^{9}$

Pineda et al. reported a case with the left atrial myxoma and acute inferolateral myocardial infarction. In the coronary angiographic examination, they found acute occlusion of the first obtuse marginal branch of the LCx. The other coronary arteries were normal without plaque or dissection, coronary aspiration yielded no thrombus, and thus they considered the etiology of occlusion as embolism rather than atherosclerosis. They performed PTCA to treat the patient as in our case. Our case also didn't have any significant lesions in other coronary arteries; additionally, the subject didn't have any ulceration or dissection. We did not apply aspiration for the subject due to short of time so we couldn't analyse the occlusion material. ${ }^{10}$ 
We couldn't perform transesophageal echocardiography or magnetic resonance imaging to confirm the diagnosis due to the emergency setting. However, transthoracic echocardiography was well enough to manage the case in the acute setting.

In conclusion, we presented a demonstrative case of myxoma with acute myocardial infarction and severe pulmonary hypertension. The patient was treated with PTCA without stenting followed by total surgical excision. Additionally, significant improvement was observed at the follow-up.

\section{Funding}

None.

\section{Competing interests}

None declared.

\section{References}

1. Bortolotti V, Maraglino G, Rubino M. Surgical excision of intracardiac myxomas: A 20-year follow-up. Ann Thorac Surg 1990;49(3):449-453.
2. Meller J, Teichholz LE, Pickard AD. Left ventricular myxoma: Echocardiographic diagnosis and review of the literature. Am J Med 1977;63(5):816.

3. Yavuz T, Peker O, Ocal A, Ibrisim E. Left atrial myxoma associated with acute myocardial infarction. Int J Cardiovasc Imaging 2005;21(2-3):235-238.

4. Lehrman KL, Prozan GB, Ullyot D. Atrial myxoma presenting as acute myocardial infarction. Am Heart J 1985;1 10(6):1293-1295.

5. Pinede L, Duhaut P, Loire R. Clinical presentation of left atrial cardiac myxoma. A series of 112 consecutive cases. Medicine (Baltimore) 2001;80(3):159-172.

6. Nakano T, Mayumi H, Hisahara M, Yasui H, Tokunaga K. The relationship between functional class, pulmonary artery pressure and size in left atrial myxoma. Cardiovasc Surg 1996;4(3):320-323.

7. Gošev I, Paić F, Durić Z, Gošev M, Ivčević S, Jakuš FB, Biočina B. Cardiac myxoma the great imitators: Comprehensive histopathological and molecular approach. Int J Cardiol 2013;164(1):7-20.

8. Zhang M, Ding L, Liu Y, Xue L Cardiac myxoma with glandular elements: A clinicopathological and immunohistochemical study of five new cases with an emphasis on differential diagnosis. Pathol Res Pract 2014;210(1):55-58.

9. Yuan SM. Cardiac myxoma: A rare cause of acute myocardial infarction. Turk Gogus Kalp Dama 2016;24(1):166-172.

10. Pineda AM, Mihos CG, Nascimento FO, Santana O, Lamelas J, Beohar N. Coronary embolization from a left atrial myxoma containing malignant lymphoma cells. Tex Heart Inst J 2015;42(6):565-568. 